\title{
ON THE NATURE OF THE TORUS IN THE COMPLEX LORENZ EQUATIONS*
}

\author{
A. C. FOWLER $\dagger$ AND M. J. McGUINNESS $\ddagger$
}

\begin{abstract}
The complex Lorenz equations are a nonlinear fifth-order set of physically derived differential equations which exhibit an exact analytic limit cycle which subsequently bifurcates to a torus. In this paper we build upon previously derived results to examine a connection between this torus at high and low $r_{1}$ (bifurcation parameter) and between zero and nonzero $r_{2}$ (complexity parameter); in so doing, we are able to gain insight on the effect of the rotational invariance of the system, and on how extra weak dispersion $\left(r_{2} \neq 0\right)$ affects the chaotic behavior of the real Lorenz system (which describes a weakly dissipative, dispersive instability).
\end{abstract}

1. Introduction. Very recently, Gibbon and McGuinness [4] have shown that a fifth-order differential system of amplitude equations may be derived from weakly dissipative systems which exhibit a primarily dispersive instability, if extra ("detuning") dispersive effects are present. Moreover, when spatial variations are excluded, this system may be viewed as a complex generalization of the Lorenz equations [11]. A concise summary of this derivation, and of other relevant papers, is given in the article by Gibbon [3]. The complex equations may be written:

$$
\dot{x}=-\sigma x+\sigma y, \quad \dot{y}=(r-z) x-a y, \quad \dot{z}=\frac{1}{2}\left(x y^{*}+x^{*} y\right)-b z,
$$

where $\sigma, b$ are real parameters, but $r=r_{1}+i r_{2}$ and $a=1-i e$ are complex, and hence $x$ and $y$ are complex also. Equations (1.1) can describe (codimension two) bifurcation behavior in baroclinic instability and nonlinear optics, and (in principle) in a variety of other physical systems too. For this reason, and also because they may increase understanding of the chaotic behavior of the real Lorenz model, a study of their solutions is of some interest.

An initial such study was made by Fowler et al. [1], and we here summarize their main findings. There is a trivial solution of (1.1), namely $x=y=z=0$, representing the state of rest in the original physical system. If we adopt $r_{1}$ as the bifurcation parameter, then this solution loses stability to a limit cycle as $r_{1}$ increases through

$$
r_{1 c}=1+\frac{\left(e+r_{2}\right)\left(e-\sigma r_{2}\right)}{(\sigma+1)^{2}}
$$

this limit cycle is the complex generalization of the nontrivial steady states in the real Lorenz equations: moreover, the limit cycle has an exact analytic form, given by

$$
x=A e^{i \omega t}, \quad y=\left(1+\frac{i \omega}{\sigma}\right) A e^{i \omega t}, \quad z=|A|^{2} / b
$$

where

$$
\omega=\frac{\sigma\left(e+r_{2}\right)}{\sigma+1}, \quad|A|^{2}=b\left(r_{1}-r_{1 c}\right),
$$

and we suppose $\omega \neq 0$. The exact limit cycle exists by virtue of the rotational invariance of the system, i.e. the equations are invariant under the transformation $x \rightarrow x e^{i \alpha}$, $y \rightarrow y e^{i \alpha}, z \rightarrow z$. As a result of this, a transformation to the rotating frame, in which

* Received by the editors February 11, 1983.

† Department of Mathematics, Massachusetts Institute of Technology, Cambridge, Massachusetts 02139.

$\ddagger$ Department of Applied Mathematics, California Institute of Technology, Pasadena, California 91125 . 
coordinates $(X, Y, Z)$ are given by

$$
x=X e^{i \omega t}, \quad y=Y e^{i \omega t}, \quad z=Z
$$

reduces the limit cycle to a continuum of fixed points, whose stability and bifurcation may be analyzed in a standard manner. We find that the limit cycle loses stability oscillatorily at a value $r_{1}=r_{l c}^{\prime}$, and solutions must (generally) bifurcate to a torus (Joseph [7]). The toroidal motion can be very nicely visualized in the rotating frame, since near bifurcation the unstable oscillation precesses slowly round the continuum of fixed points (underlying limit cycle) (Fowler et al. [1]). Numerically, we find for $e=3 r_{2}, \sigma=2$ that the bifurcation to a torus is subcritical for $0<r_{2} \leqslant 1$; this is analogous to the subcritical Hopf bifurcation in the Lorenz model (McLaughlin and Martin [13], Roshchin [20]). However, whereas the resultant motion tends to be aperiodic in the real Lorenz system when the nontrivial fixed points are unstable, numerical evidence when $r_{2} \neq 0$ suggests that the bifurcating branch might "bend back" so that one sees snap-through instability to a stable torus on the upper branch.

This is the kind of behavior one would associate with the Stuart-Landau type amplitude equation

$$
\frac{d A}{d \tilde{t}}=k_{1} A+k_{2}|A|^{2} A+k_{3}|A|^{4} A
$$

where $\tilde{t}$ is a slow time, and $A$ the complex slowly-varying amplitude of an $e^{i \Omega t}$ disturbance to the underlying limit cycle. For the complex equations, one analytically derives (1.6) (but without $k_{3}$ ) by the method of multiple scales. Subcriticality suggests $\operatorname{Re}\left(k_{2}\right)>0$. However, if (numerically) $0<\operatorname{Re}\left(k_{2}\right) \ll 1$, then we would formally derive (1.6) using the method of multiple scales by examining the distinguished limit where the amplitude $\varepsilon$ is such that

$$
\operatorname{Re}\left(k_{2}\right) \sim \varepsilon^{2}, \quad\left|r_{1}-r_{1 c}^{\prime}\right| \sim \varepsilon^{4}, \quad \tilde{t}=\varepsilon^{4} t,
$$

and if $\operatorname{Re}\left(k_{3}\right)<0$, the upper branch would be stable. Though we have not even computed $k_{2}$, let alone $k_{3}$, the numerical form of the solutions could plausibly suggest that the bifurcation analysis does (at least qualitatively) carry "round the corner" in this way.

The form of the bifurcating torus is best represented using the five real variables $x_{1}, x_{2}, \cdots, x_{5}$, where

$$
x=|A|\left(x_{1}+i x_{2}\right), \quad y=|A|\left(x_{3}+i x_{4}\right), \quad z=\left(|A|^{2} / b\right) x_{5} .
$$

The equations may then be written

$$
\dot{\mathbf{x}}=\mathbf{f}(\mathbf{x}),
$$

and the property of rotational invariance follows from

$$
\mathbf{f}(R \mathbf{x})=R \mathbf{f}(\mathbf{x}),
$$

where $R(\alpha)$ is the real five-by-five matrix corresponding to the change of variables $(x, y, z,) \rightarrow\left(x e^{i \alpha}, y e^{i \alpha}, z\right)$. The transformation (1.8) locates the limit cycle as

$$
\mathbf{x}=R(\omega t) \mathbf{x}_{0}, \quad \mathbf{x}_{0}=(1,0,1, \omega / \sigma, 1)^{T} ;
$$

for $r_{1}$ close to $r_{1 c}^{\prime}$, the fixed point $\mathbf{x}_{0}$ in the rotating frame becomes oscillatorily unstable with eigenfrequency $\Omega$, and corresponding eigenvector (in the fixed frame) $R(\omega t) \mathbf{U}_{\Omega}$, where $\mathbf{U}_{\Omega}$ does not depend on time. The multiple scales analysis then shows that the 
form of the bifurcating torus is given by

$$
\mathbf{x}=R(\tilde{\omega} t) \mathbf{u}(\tilde{\Omega} t),
$$

where for $\left|r_{1}-r_{1 c}^{\prime}\right| \ll 1\left(\sim \varepsilon^{2}\right)$, we can write

$$
\begin{aligned}
& \tilde{\omega}=\omega+O\left(\varepsilon^{2}\right), \\
& \tilde{\Omega}=\Omega+O\left(\varepsilon^{2}\right), \\
& \mathbf{u}=\mathbf{x}_{0}+\varepsilon\left\{\mathbf{U}_{\Omega} e^{i \tilde{\Omega} t}+(*)\right\}+O\left(\varepsilon^{2}\right),
\end{aligned}
$$

and $\varepsilon$ is a measure of the amplitude. The notation in (1.12) signifies $R(\theta)$ and $\mathbf{u}(\theta)$ are $2 \pi$-periodic functions of $\theta$. As discussed, we surmise that the form of (1.12) qualitatively represents the upper branch stable torus which is numerically observed. The "bifurcation theory" version of (1.12) and (1.13) has recently been given by Renardy [17]; see also Rand [16].

Various questions now present themselves. Since the form of (1.12) is representative of the rotational invariance of the system, we may inquire whether this form persists for larger $r_{1}$. If we examine phase plots of $x_{1}$ versus $x_{2}$, or $x_{3}$ versus $x_{4}$ (i.e. $\operatorname{Re} x / \operatorname{Im} x$ or $\operatorname{Re} y / \operatorname{Im} y$ ), the limit cycle will appear as a circle (because of the form of $R$ ) and the bifurcating torus will look like the projection of a solenoid (or a trajectory wrapped round a doughnut). Figure 1 shows that this behavior persists away from $r_{1 c}^{\prime}$, and is suggestive of (1.12) - a limit cycle being precessed around the origin.

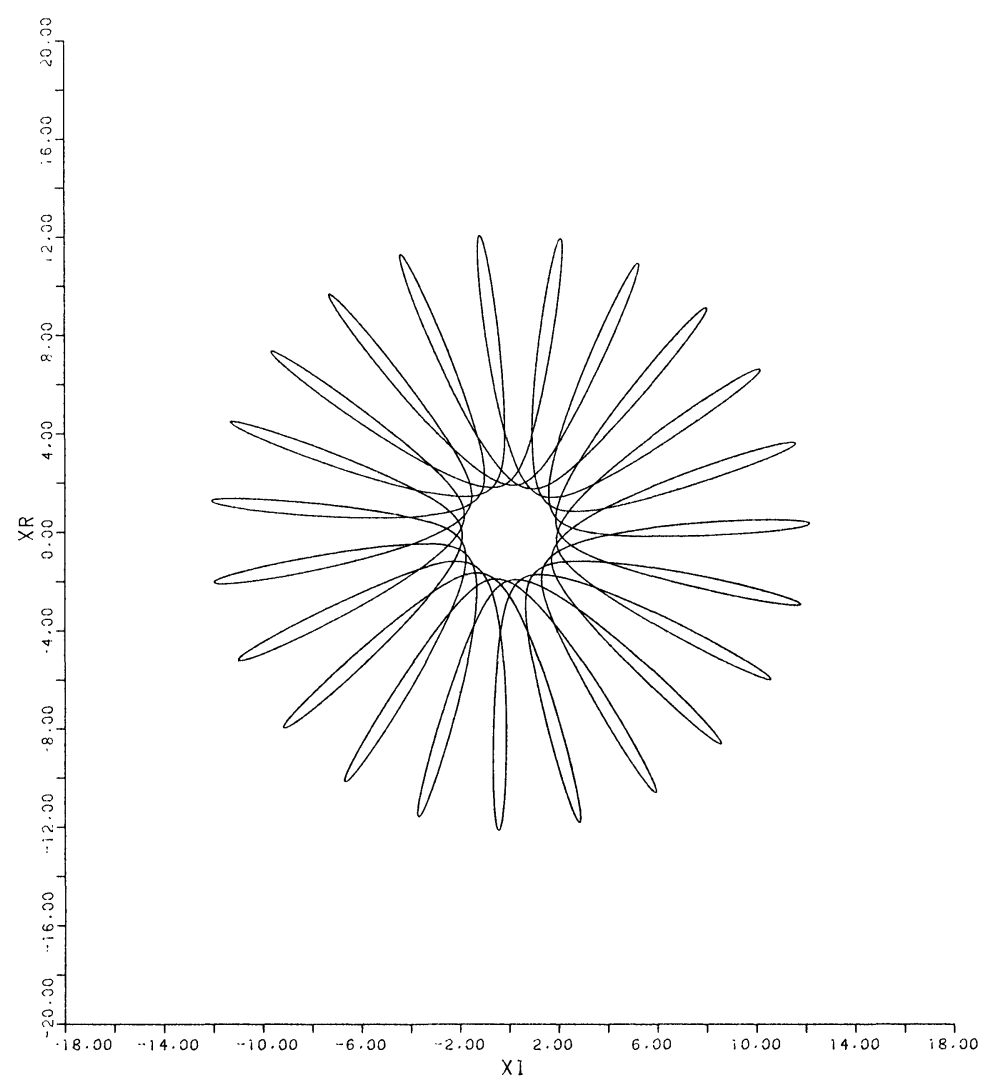

FIG. 1. Numerical solution of the complex Lorenz equations at $b=0.8, \sigma=2, r_{1}=70, r_{2}=0.4, e=1.2$. 
The particular form of $R$ (given by Fowler et al. [1]) shows that for solutions of the form (1.12), $x_{5}$ will be simply periodic of period $\tilde{\Omega}$, whereas the other $x_{i}$ will be the sum of terms of the form $e^{ \pm i \tilde{\omega} t} \times$ function of period $\tilde{\Omega}$. Spectral analysis of $x, y$, and $z$ will therefore reveal (if $(1.12)$ is relevant) peaks at $\tilde{\Omega}, 2 \tilde{\Omega}, \cdots$ for $z\left(x_{5}\right)$, and peaks at $|n \tilde{\Omega} \pm \tilde{\omega}|, n=0,1, \cdots$ for $x$ and $y\left(x_{1}, \cdots, x_{4}\right)$. We can also use this to check (1.12), and indeed we do find that spectral plots are consistent with this description.

This then raises a further question: if (1.12) is a valid description of the torus for all $r_{1}$, how do the frequencies change as $r_{2} \rightarrow 0$, since toroidal motions of this type do not (and cannot) occur in the real Lorenz system (Ott [14])? Particularly, how does the torus adjust itself as $r_{2} \rightarrow 0$ at high $r_{1}$, where the real Lorenz system has a stable periodic solution (Robbins [19])! In fact, a curious thing happens: as $r_{2} \rightarrow 0$ at $r_{1} \gg 1$, we find that if $\tilde{\omega} \rightarrow \tilde{\omega}_{0}$ and $\tilde{\Omega} \rightarrow \tilde{\Omega}_{0}$, then $\tilde{\omega}_{0}=\tilde{\Omega}_{0} / 2$. The motion becomes periodic because the "splitting" frequency $\tilde{\omega}$ locks on to half the rotating limit cycle frequency $\tilde{\Omega}$. The periodic motion then has frequency $\tilde{\omega}_{0}$, and can be written

$$
\mathbf{x}=R\left(\tilde{\omega}_{0} t\right) \mathbf{u}\left(2 \tilde{\omega}_{0} t\right)
$$

(if (1.12) is valid). Spectrally, we will see peaks for $x$ and $y$ (now real variables) at $\tilde{\omega}_{0}, 3 \tilde{\omega}_{0}, 5 \tilde{\omega}_{0}$, etc., whereas $z$ has frequencies $2 \tilde{\omega}_{0}, 4 \tilde{\omega}_{0}, \cdots$. This simply represents

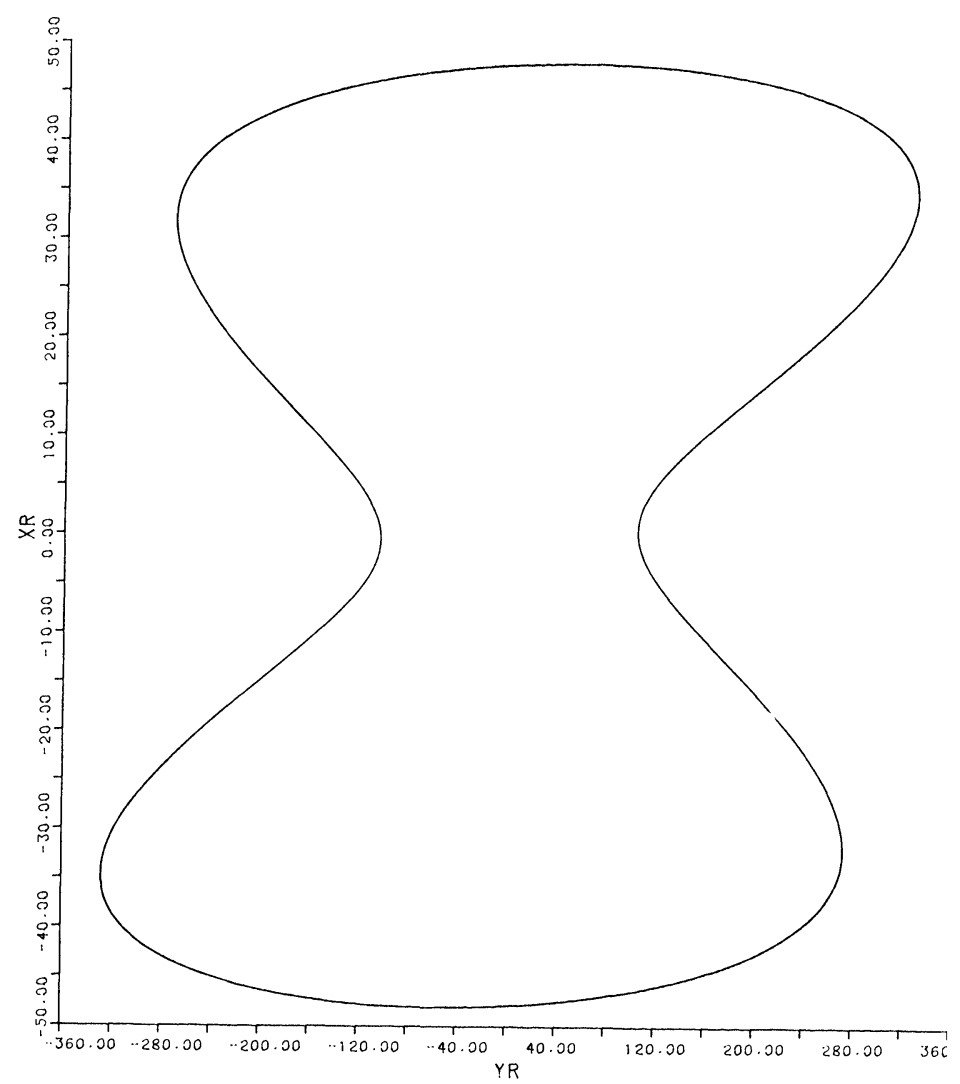

FIG. 2. (a) $X_{R}-X_{R}$ plot at $r_{1}=1000, r_{2}=0, e=0, b=0.8, \sigma=2$. 
the natural symmetry of the real Lorenz equations under the transformation $(x, y, z) \rightarrow$ $(-x,-y, z)$.

A little thought shows that as $r_{2} \rightarrow 0$ at high $r_{1},\left(x_{R}, y_{R}\right)$ plots should trace out a torus whose envelope is the familiar $(x, y)$ plot of the Robbins limit cycle. Similarly, a $\left(y_{R}, z\right)$ plot will represent the Robbins $(y, z)$ phase plot rotating round a line parallel to the $z$-axis (her $w$-axis). These considerations are borne out in Figs. 2 and 3, computed at $r_{1}=1000$, and $r_{2}=0,1$. Note that the envelopes of the complex case do not exactly overlie the (real) limit cycle.

In the next section we follow up the ideas presented here, by extending the analysis at large $r_{1}$ of the real Lorenz equations to the complex case, thereby obtaining an analytic description of a nonlinear torus. In addition, we may hope to gain some insight into how the motion on a torus can break down into chaos. It will be seen that the analysis for $\S 2$ relies heavily on previous work by Robbins [19] and particularly on more recent work of Sparrow [21].

2. A nonlinear torus at high $\boldsymbol{r}_{\mathbf{1}}$. We consider the equations

$$
\dot{x}=-\sigma x+\sigma y, \quad \dot{y}=(r-z) x-a y, \quad \dot{z}=\frac{1}{2}\left(x y^{*}+x^{*} y\right)-b z,
$$

where $r=r_{1}+i r_{2}, a=1-i e$, in the limit $r_{1} \rightarrow \infty$, where a dot denotes differentiation

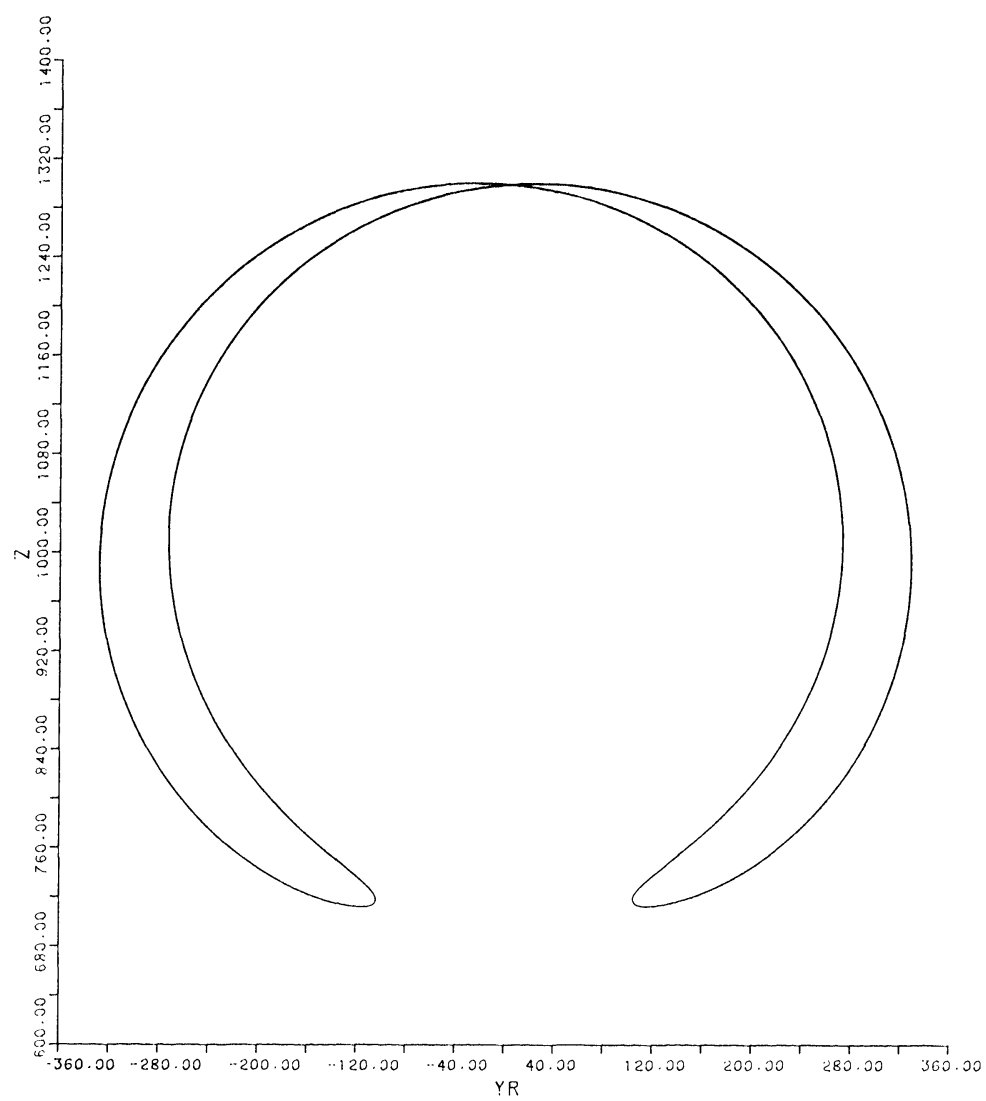

FIG. 2. (b) $Y_{R}-Z$ plot at $r_{1}=1000, r_{2}=0, e=0, b=0.8, \sigma=2$. 


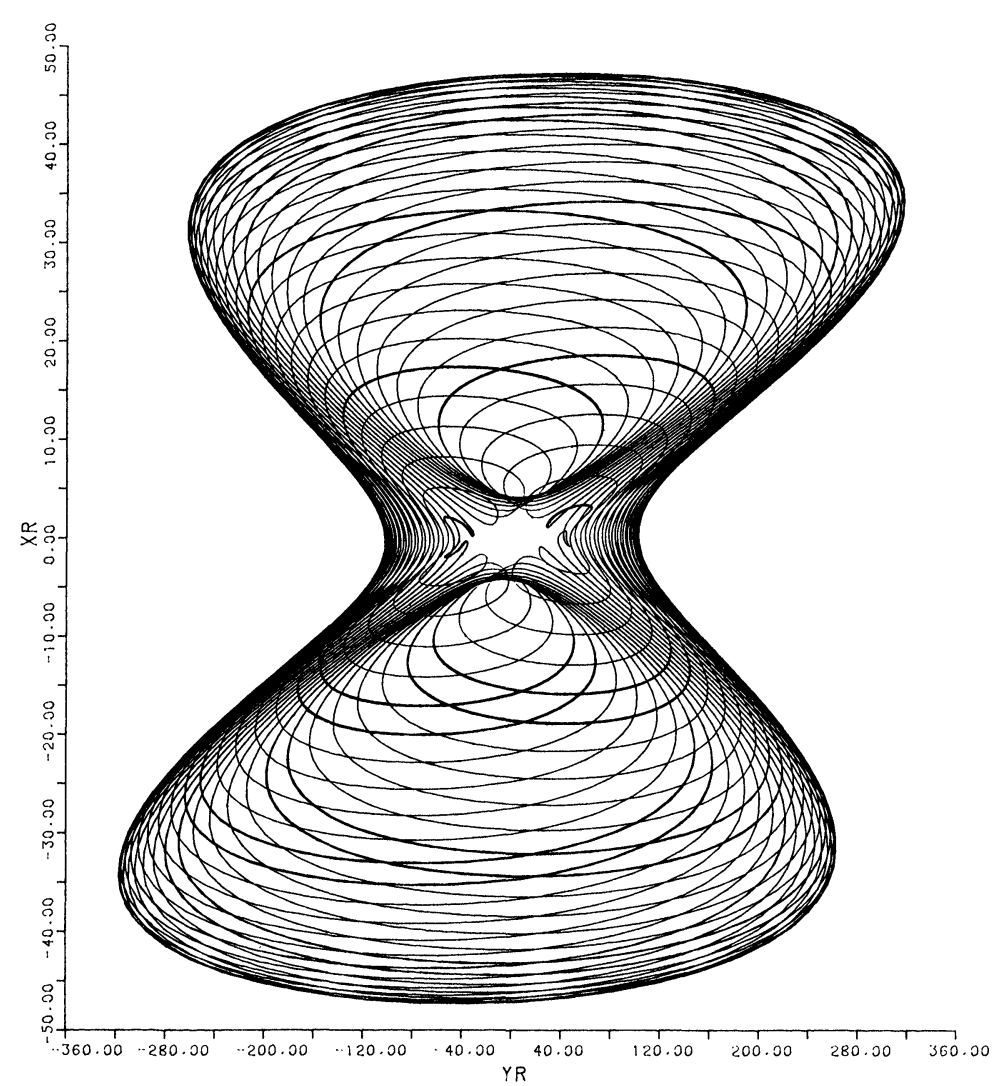

FIG. 3. (a) $X_{R}-Y_{R}$ plot at $r_{1}=1000, r_{2}=1, e=3, b=0.8, \sigma=2$.

with respect to time $T$. To isolate the nature of the torus in this limit, we define

$$
\varepsilon=\frac{1}{\sqrt{r_{1} \sigma}}, \quad r_{2}=\sqrt{\frac{r_{1}}{\sigma}} \rho_{2}, \quad e=\sqrt{r_{1} \sigma} a_{2},
$$

rescale the variables as

$$
x=\sqrt{r_{1} \sigma} \xi, \quad y=r_{1} \eta, \quad z=r_{1} \zeta, \quad T=\frac{1}{\sqrt{r_{1} \sigma}} t,
$$

and consider the asymptotic solutions of the resulting equations in the limit where $\varepsilon \rightarrow 0, \sigma, \rho_{2}, a_{2}, b \sim O(1)$ :

$$
\begin{aligned}
& \dot{\xi}=\eta-\varepsilon \sigma \xi, \\
& \dot{\eta}=(1-\zeta) \xi+i a_{2} \eta+\varepsilon\left[i \rho_{2} \xi-\eta\right], \\
& \dot{\zeta}=\frac{1}{2}\left(\xi \eta^{*}+\xi^{*} \eta\right)-\varepsilon b \zeta .
\end{aligned}
$$

(Here a dot indicates $d / d t$.)

This rescaling is standard; we only comment on the definition of $r_{2}$ and $e$. We choose $r_{2}, e \sim \sqrt{r_{1}}$, so that the damping term due to $r_{2}$ is absent at $O(1)$, but present at $O(\varepsilon)$ : otherwise (if present at $O(1))$ the system is not weakly dissipative $\left(r_{1}\right.$ is not high enough): if absent at $O(\varepsilon), r_{2}$ is irrelevant to the dynamics. (2.4) is therefore an interesting distinguished limit. Similarly, it is natural to scale $e$ so that its (rotational) 


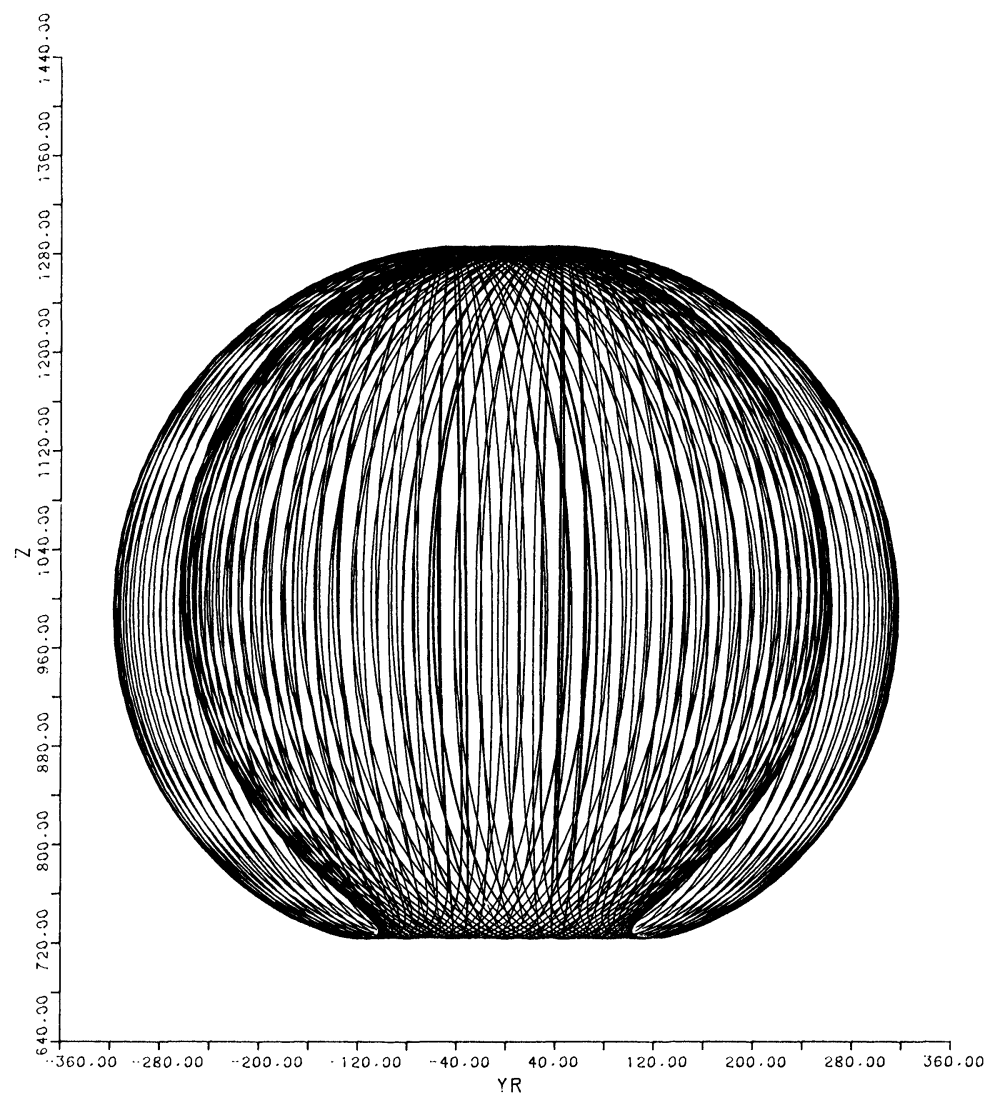

FIG. 3. (b) $Y_{R}-Z$ plot at $r_{1}=1000, r_{2}=1, e=3, b=0.8, \sigma=2$.

effect is felt at $O(1)$. This discussion incidentally suggests that there may be some critical value of $\rho_{2}$, beyond which the torus (and the ensuing analysis) does not exist: we note for the moment that this is consistent with curve $B$ in Fig. 3 of Fowler et al. [1].

From (2.4), we derive

$$
\begin{aligned}
& \frac{1}{2} \frac{d}{d t}\left[w^{2}+|\eta|^{2}\right]=\varepsilon\left[b w(1-w)+\frac{1}{2} i \rho_{2}\left(\xi \eta^{*}-\xi^{*} \eta\right)-|\eta|^{2}\right] \\
& \frac{d}{d t}\left[w+\frac{1}{2}|\xi|^{2}\right]=\varepsilon\left[b(1-w)-\sigma|\xi|^{2}\right]
\end{aligned}
$$

where we have put $w=1-\zeta$. To solve (2.4), we now employ the method of averaging. ${ }^{1}$ We define a slow time

$$
\tau=\varepsilon t
$$

then (2.5) implies

$$
w^{2}+|\eta|^{2}=B^{2}(\tau), \quad w+\frac{1}{2}|\xi|^{2}=D(\tau)
$$

${ }^{1}$ A more methodical procedure is to use the method of Kuzmak-Luke-Kogelman-Keller [8], [9], [10], [12], [15], but at leading order, the two methods are equivalent. 
correct to $O(\varepsilon)$. This implies that we can define functions $\theta, \phi$ (of $t$ and $\tau$ ) such that $(2.8)_{1,2}$ $w=-B \cos \theta, \quad \eta=B e^{i \phi} \sin \theta$,

and hence from $(2.4)_{2}$

$$
\xi=\left[\dot{\eta}-i a_{2} \eta\right] / w+O(\varepsilon)=-e^{i \phi}\left[\dot{\theta}+i\left(\dot{\phi}-a_{2}\right) \tan \theta\right]
$$

correct to $O(\varepsilon) .(2.7)_{2}$ then implies that

$$
D+B \cos \theta=\frac{1}{2}\left[\dot{\theta}^{2}+\left(\dot{\phi}-a_{2}\right)^{2} \tan ^{2} \theta\right] .
$$

From $(2.4)_{1}, \dot{\xi}=\eta+O(\varepsilon)$, whence (2.8) implies, on equating imaginary parts and integrating, that

$$
\left(\dot{\phi}-a_{2}\right) \tan \theta=\frac{G(\tau)+a_{2} \cos \theta}{\sin \theta}
$$

(the real part just leads back to (2.9)). (2.9) now implies that $\theta$ satisfies

$$
\frac{1}{2} \dot{\theta}^{2}=D+B \cos \theta-\frac{\left[G+a_{2} \cos \theta\right]^{2}}{2 \sin ^{2} \theta},
$$

$\dot{\phi}$ is given by (2.10), and in terms of $\theta$ and $\phi$,

$$
\begin{aligned}
\xi & =-e^{i \phi}\left[\dot{\theta}+i\left\{\frac{G+a_{2} \cos \theta}{\sin \theta}\right\}\right], \\
\eta & =B e^{i \phi} \sin \theta, \\
w & =-B \cos \theta .
\end{aligned}
$$

The difference between real and complex cases is in $G$. In the real case, $G=a_{2}=0$, and (2.11) describes a simple pendular motion on the circle $w^{2}+\eta^{2}=$ constant. When $G \neq 0, a_{2} \neq 0$, the motion is pendular on the sphere $w^{2}+|\eta|^{2}=$ constant. Observing that the kinetic energy of rotation (divided by $B^{2}$, or using $1 / B$ as a time unit) is $\left(\dot{\phi}^{2} \sin ^{2} \theta\right) / 2$, and that from (2.10), $\dot{\phi}=\left[G \cos \theta+a_{2}\right] / \sin ^{2} \theta$, it follows that (2.11) may be written in the form

$$
\frac{1}{2} \dot{\theta}^{2}+\frac{1}{2} \dot{\phi}^{2} \sin ^{2} \theta-B \cos \theta=D+\frac{1}{2}\left(a_{2}^{2}-G^{2}\right),
$$

which is the statement of conservation of kinetic and potential energy for this system. This illuminates the avoidance of the states of rest, $\theta=0, \pi$, as is evident in Figs. 1 and $3 \mathrm{a}$.

To solve (2.11) we must have $D>-B$. Then one easily finds that

$$
t=\int_{\cos \theta} \frac{d u}{\left[(2 D+2 B u)\left(1-u^{2}\right)-\left(G+a_{2} u\right)^{2}\right]^{1 / 2}} .
$$

The cubic inside the square root has zeros $u_{1}, u_{2}, u_{3}$ such that

$$
1>u_{1}>0>u_{2}>-1>u_{3},
$$

(provided $G$ is small enough); the square root is defined for $u_{1}>u>u_{2}$, and for such $u$, we have

$$
\begin{aligned}
\sqrt{2 B} t+\text { constant }(\text { the phase }) & =\int_{\cos \theta}^{u_{1}} \frac{d u}{\left[\left(u_{1}-u\right)\left(u-u_{2}\right)\left(u-u_{3}\right)\right]^{1 / 2}} \\
& =\frac{2}{\left(u_{1}-u_{3}\right)^{1 / 2}} F(\lambda, p)
\end{aligned}
$$


where

$$
\lambda=\sin ^{-1}\left(\frac{u_{1}-\cos \theta}{u_{1}-u_{2}}\right)^{1 / 2}, \quad p=\left(\frac{u_{1}-u_{2}}{u_{1}-u_{3}}\right)^{1 / 2},
$$

and

$$
F(\lambda, p)=\int_{0}^{\lambda} \frac{d \alpha}{\left[1-p^{2} \sin ^{2} \alpha\right]^{1 / 2}}
$$

is the elliptic integral of the first kind. ${ }^{2}$

Defining

$$
u=\sqrt{\frac{B\left(u_{1}-u_{3}\right)}{2}} t+\text { arbitrary phase }
$$

we have

$$
\frac{u_{1}-\cos \theta}{u_{1}-u_{2}}=\operatorname{sn}^{2} u, \quad k^{2}=\left(\frac{u_{1}-u_{2}}{u_{1}-u_{3}}\right)
$$

where $k$ is the modulus: hence we derive expressions for $\xi, \eta, w$ (at leading order) in terms of elliptic functions: particularly,

$$
\begin{aligned}
& w=B\left[-u_{3}-\left(u_{1}-u_{3}\right) d n^{2} u\right], \\
& \sin \theta=\left[1-u_{3}^{2}+\left(u_{1}-u_{3}\right) d n^{2} u\left\{\left(u_{1}-u_{2}\right) s n^{2} u-\left(u_{1}+u_{3}\right)\right\}\right]^{1 / 2}, \\
& \dot{\theta}=\frac{\sqrt{2 B\left(u_{1}-u_{3}\right)}\left(u_{1}-u_{2}\right) s n u c n u d n u}{\left[1-u_{3}^{2}+\left(u_{1}-u_{3}\right) d n^{2} u\left\{\left(u_{1}-u_{2}\right) s n^{2} u-\left(u_{1}+u_{3}\right)\right\}\right]^{1 / 2}} .
\end{aligned}
$$

We do not need to consider the explicit forms for $\xi, \eta$ and $w$ further, beyond observing that indeed $\xi$ and $\eta$ are generally doubly periodic and $w$ is singly periodic. We come back to the form of (2.12) in due course.

In order to determine evolution equations for $B, D$ and $G$, we require three "near"'-conservation laws for these variables. Two such are (2.5) and the third follows from

$$
\frac{d}{d t}\left[\xi \eta^{*}-\xi^{*} \eta-2 i a_{2} w\right]=\varepsilon\left[-(\sigma+1)\left(\xi \eta^{*}-\xi^{*} \eta\right)-2 i \rho_{2}|\xi|^{2}-2 i a_{2} b(1-w)\right]
$$

To $O(\varepsilon), \xi, \eta, w$ are given by (2.12), and the right-hand sides of (2.5) and (2.12) are determined accordingly. We find that

$$
\begin{aligned}
& \xi \eta^{*}-\xi^{*} \eta=2 i B G+2 i a_{2} w \\
& |\eta|^{2}=B^{2} \sin ^{2} \theta \\
& |\xi|^{2}=\dot{\theta}^{2}+\left\{\frac{G+a_{2} \cos \theta}{\sin \theta}\right\}^{2}
\end{aligned}
$$

${ }^{2}$ Actually, the phase in $(2.15)$ is a slowly varying function of time, whose computation is extremely tortuous. Fortunately, it is immaterial to the determination of the slowly varying amplitudes $B, D, G$. 
and it follows that, to $O(\varepsilon)$,

$$
\begin{gathered}
\frac{1}{2} \frac{d}{d t}\left[B^{2}\right]=\varepsilon\left[-b B \cos \theta\{1+B \cos \theta\}+\rho_{2} B G+a_{2} \rho_{2} B \cos \theta-B^{2} \sin ^{2} \theta\right] \\
\frac{d D}{d t}=\varepsilon\left[b(1+B \cos \theta)-\sigma\left\{\dot{\theta}^{2}+\left(\frac{G+a_{2} \cos \theta}{\sin \theta}\right)^{2}\right\}\right] \\
\frac{d}{d t}(B G)=\varepsilon\left[-(\sigma+1)\left(B G+a_{2} B \cos \theta\right)\right. \\
\left.+\rho_{2}\left\{\dot{\theta}^{2}+\left(\frac{G+a_{2} \cos \theta}{\sin \theta}\right)^{2}\right\}+a_{2} b(1+B \cos \theta)\right]
\end{gathered}
$$

where $\theta$ is given by (2.19). Now $s n^{2} u$ has period $2 K$ in $u$, where $K$ is the complete elliptic integral of the first kind, $K=\int_{0}^{\pi / 2}\left[1-k^{2} \sin ^{2} \alpha\right]^{-1 / 2} d \alpha$, and hence a period in $t$ of $2 K\left[2 / B\left(u_{1}-u_{3}\right)\right]^{1 / 2}=T$, say. Therefore the right-hand sides of (2.23) are (considering $B, D, G$ nearly constant) approximately periodic, and so if we integrate over a time interval $(t, t+T)$, we find (for example)

$$
\frac{1}{T} \int_{t}^{t+T} \frac{d D}{d t} d t=\frac{1}{T}[D(\tau+\varepsilon T)-D(\tau)] \approx \varepsilon D^{\prime}(\tau)=\varepsilon \overline{[\cdot]},
$$

where the overbar denotes the average value of the square-bracketed term over a period of the elliptic functions. We note from (2.11) that

$$
\overline{\dot{\theta}^{2}+\left\{\frac{G+a_{2} \cos \theta}{\sin \theta}\right\}^{2}}=2[D+B \overline{\cos \theta}]
$$

hence the average equations for $B, D, G$ are simply

$$
\begin{aligned}
& \frac{1}{2} \frac{d}{d \tau}\left(B^{2}\right)=-b B c_{1}-b B^{2} c_{2}+\rho_{2} B G+a_{2} \rho_{2} B c_{1}-B^{2}\left(1-c_{2}\right), \\
& \frac{d D}{d \tau}=b\left(1+c_{1} B\right)-2 \sigma\left(D+c_{1} B\right), \\
& \frac{d}{d \tau}(G B)=-(\sigma+1)\left(B G+a_{2} B c_{1}\right)+2 \rho_{2}\left(D+c_{1} B\right)+a_{2} b\left(1+c_{1} B\right),
\end{aligned}
$$

where

$$
c_{1}=\overline{\cos \theta}, \quad c_{2}=\overline{\cos ^{2} \theta}
$$

These averages are computed in terms of elliptic integrals from (2.19), and one finds

$$
\begin{aligned}
& c_{1}=u_{3}+\left(u_{1}-u_{3}\right) \frac{E}{K} \\
& c_{2}=\left[u_{3}^{2}-\frac{1}{3}\left(u_{1}-u_{3}\right)^{2}\left(1-k^{2}\right)\right]+2\left(u_{1}-u_{3}\right) \frac{E}{K}\left[u_{3}+\frac{1}{3}\left(u_{1}-u_{3}\right)\left(2-k^{2}\right)\right]
\end{aligned}
$$

where $E$ is the complete elliptic integral of the second kind, $E=$ $\int_{0}^{\pi / 2}\left(1-k^{2} \sin ^{2} \alpha\right)^{1 / 2} d \alpha$, and here $k^{2}=\left(u_{1}-u_{2}\right) /\left(u_{1}-u_{3}\right)$. The equations $(2.26)$ may 
finally be written in the form

$$
\begin{aligned}
& D^{\prime}=b\left(1+c_{1} B\right)-2 \sigma\left(D+c_{1} B\right), \\
& B^{\prime}=\rho_{2} \Gamma / B-\left(b-a_{2} \rho_{2}\right) c_{1}-\left[1-(1-b) c_{2}\right] B, \\
& \Gamma^{\prime}=-(\sigma+1)\left(\Gamma+a_{2} c_{1} B\right)+2 \rho_{2}\left(D+c_{1} B\right)+a_{2} b\left(1+c_{1} B\right),
\end{aligned}
$$

where we define

$$
\Gamma=B G
$$

\section{The nature of the solutions.}

Steady states. Consider the equations (2.30). These are defined for $B>0, D>-B$ (at least when $G=0$; more strictly $D+B u_{1}>0$ ). We define

$$
\begin{aligned}
& U=D / B, \quad c=-c_{1}=-u_{3}-\left(u_{1}-u_{3}\right)(E / K), \\
& \phi(u)=(U+u)\left(1-u^{2}\right)-\gamma(1+\alpha u)^{2}, \\
& \gamma=G^{2} / 2 B, \quad \alpha=a_{2} / G,
\end{aligned}
$$

so that $u_{1}>u_{2}>u_{3}$ are the roots of $\phi=0$, and hence

$$
\begin{aligned}
& u_{1}+u_{2}+u_{3}=-U-\gamma \alpha^{2}, \\
& u_{2} u_{3}+u_{3} u_{1}+u_{1} u_{2}=-1+2 \gamma \alpha, \\
& u_{1} u_{2} u_{3}=U-\gamma .
\end{aligned}
$$

By substituting $E / K$ from (3.1), we find, using (3.2), that

$$
c_{2}=\frac{1}{3}[1-2 \gamma \alpha]+\frac{2}{3} c\left[U+\gamma \alpha^{2}\right] \text {. }
$$

We now seek the steady state solutions of (2.30). Using (3.1) and (3.3), we find $B, \Gamma$ are given in terms of $U$ and $c$ by

$$
\begin{gathered}
B=\frac{b}{b c+2 \sigma(U-c)} \quad\left(\text { from }(2.30)_{1}\right), \\
\Gamma=\frac{b\left[2 \rho_{2}(U-c)+a_{2}\{(\sigma+1) c+2 \sigma(U-c)\}\right]}{(\sigma+1)[b c+2 \sigma(U-c)]} \quad\left(\text { from }(2.30)_{3}\right),
\end{gathered}
$$

and then $(2.30)_{2}$ (using (3.3), (3.4) and (3.5)) gives, after some algebra,

$$
2 \lambda-1+d\left(\lambda, b, \rho_{2}, a_{2}, c, U\right)=\frac{1-c U}{3 c(U-c)},
$$

where

(3.7) $\lambda=\frac{\sigma+1}{b+2}$

$$
d=\frac{2}{c}\left[\rho_{2}-\frac{1}{3}(1-b) a_{2}\right]\left[\rho_{2}-a_{2}+\frac{1}{(b+2) \lambda}\right]\left[(2 \lambda-1)\left(\frac{U-c}{b}\right)+\frac{U}{b+2}\right]
$$

Equations (3.6) and (3.8) are really two equations for $U$ and $c$, since

$$
c=-u_{3}-\left(u_{1}-u_{3}\right) E / K=c(U, \gamma, \alpha),
$$


from (3.1). The constraints on the solutions are that

$$
\begin{aligned}
& U \geqq-1, \quad[b c+2 \sigma(U-c)]>0 \quad(B>0), \\
& 0 \leqq \gamma \leqq \gamma_{c}, \quad \lambda>0,
\end{aligned}
$$

where at $\gamma_{c}, \phi$ has a double root $u_{1}=u_{2}=u_{c}$. In practice, we pick $U$ and $c$ (satisfying (3.10)); then (3.4) and (3.5) determine $B$ and $G$ and hence $D,(3.1)$ gives $\gamma$ and we pick $a_{2}$ so that $c$ computed from (3.9) equals our chosen $c$. With given $\rho_{2}, b,(3.8)$ determines $d=d(\lambda)$, and finally (3.6) prescribes $\lambda$. Thus a two-parameter $(U, c)$ family of possible steady state solutions of (2.30) determines a two-parameter $\left(\sigma, a_{2}\right)$ family of surfaces in parameter space. We would like to know how many solutions exist, and for what values of $\sigma$ and $a_{2}$. Obviously, this is a complicated numerical problem in general, and we do not pursue it here: however, the answer is relatively easily given in the real case, when $\rho_{2}=a_{2}=0$. The details are given by Sparrow [21]; we should emphasize that the treatment above and following stems largely from his work. If $\rho_{2}=a_{2}=0$, then $G=0$ and $c=c(U)$. Also

$$
2 \lambda-1=\frac{1-c U}{3 c(U-c)}=\Psi, \text { say. }
$$

For $U>1, B>0$ and $\Psi$ decreases monotonically from 1 to $\frac{1}{3}$ as $U$ decreases from $\infty$ to 1 . Thus for each $\lambda \in\left(\frac{2}{3}, 1\right)$ there is a solution with $U>1$. For $U<1, \Psi$ increases as $U$ decreases from $\Psi=\frac{1}{3}$ at $U=1$ to $\Psi=\infty$ at $U \approx 0.65$, where $U=c(U)$. For $U \leqq 0.65$, $U-c<0$, and apparently no solutions with $\lambda>0$ are possible. This shows that for

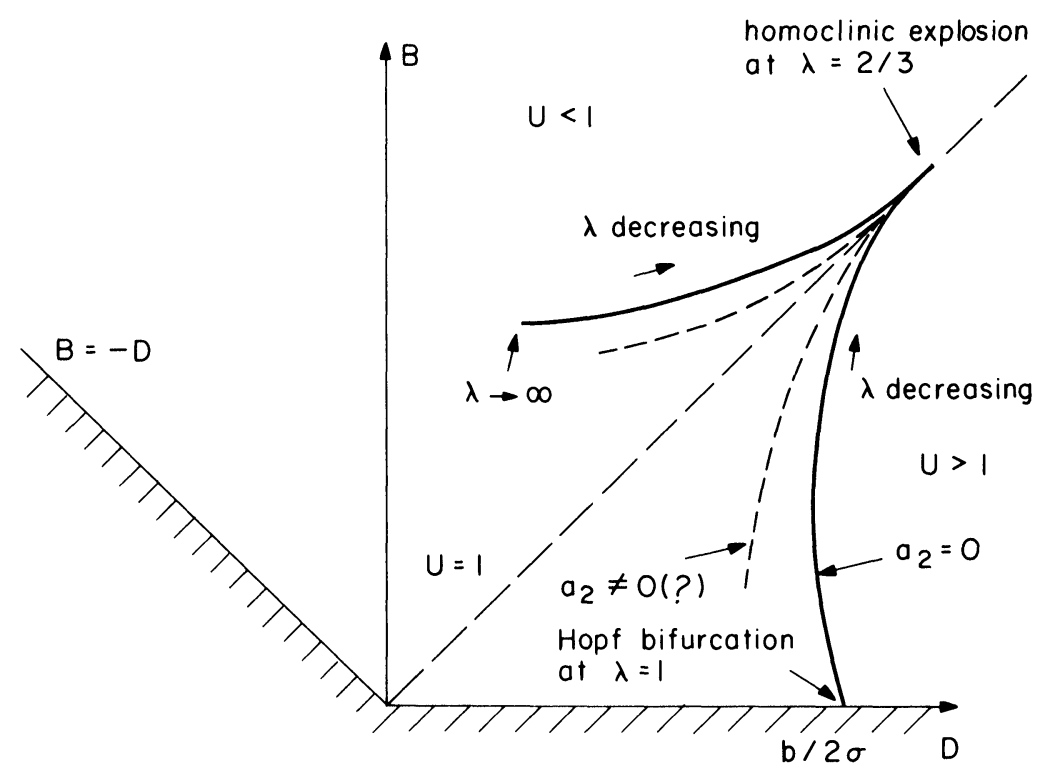

FIG. 4. Variation of equilibria of (2.30) when $a_{2}\left(=\rho_{2}\right)=0$ (solid line) and (possibly) when $a_{2} \neq 0$ (dotted line). As $\lambda$ decreases from $\infty$ to $\frac{2}{3}\left(\right.$ at $\left.\rho_{2}=0\right)$, the $U<1$ equilibrium approaches the "homoclinic explosion" at $\lambda=\frac{2}{3}$, where $B=D=1$. Locally, this approach is cusp-shaped, since (3.12), (3.15) and (3.17) describe the curve locally as $|\Delta| \sim 32 \exp [-4 / \delta]$, where $\delta$ is proportional to distance along $U=1, \Delta$ is distance from $U=1$. As $\lambda$ increases from $\frac{2}{3}$ to 1 , the $U>1$ equilibrium explodes from $B=D=1$, and reaches the Hopf-bifurcating equilibrium at $(b / 2 \sigma, 0)$ when $\lambda=1$. We may expect similar behavior for $\rho_{2} \neq 0$, as indicated by the dotted line. This figure may be compared with a similar one (energy of a simple pendulum) in Howard [6]. 
$\lambda \in\left(\frac{2}{3}, \infty\right)$ there is a solution with $U<1$. Additionally, the origin corresponds to a steady state $B=D=1$, i.e. $U=1$, and the nontrivial steady states to a steady state $B=0, D=b / 2 \sigma$.

It has been pointed out by Robbins [18] and Sparrow that the $U>1$ solution is that involved in the subcritical Hopf bifurcation (near $\lambda=1$ when $r_{1}$ is large), whereas the $U<1$ solution is the numerically observed limit cycle at large $r_{1}$. As $\lambda$ decreases through $\lambda=1$, the $U>1$ solution bifurcates from $B=0, D=b / 2 \sigma$ (the Hopf bifurcation). As $\lambda$ decreases towards $\frac{2}{3}$, the $U>1$ and $U<1$ solutions approach the line $U=1$ and coalesce at $B=D=1$ when $\lambda=\frac{2}{3}$. The coalescence at $B=D=1$ represents the approach to a homoclinic orbit; the homoclinic explosion takes place at $\lambda=\frac{2}{3}$ (for high $\left.r_{1}\right)$, and the strange invariant set is "born" at this value. The situation is represented in Fig. 4.

Of most interest as regards "chaos," is the passage of the equilibrium solution through $B=1=D$ as $U$ varies through $U=1$. Since this is directly associated with the strange attractor when $a_{2}=0$, we enquire if this coalescence can persist when $a_{2} \neq 0$. In Fig. 4 we have also drawn a schematic track of equilibria as $U$ and, say, $\lambda$ vary with, say, fixed $a_{2}$. We can imagine an orthogonal $G$-axis pointing up out of the paper. We have indicated that with $a_{2} \neq 0$ the solution track also encounters the "homoclinic" point at $B=D=1, G=a_{2}$. From (3.6) and (3.8), we can see how this can be so.

The only way $(B, D, \Gamma)$ can approach $\left(1,1, a_{2}\right)$ is if $U, c \rightarrow 1$, unless $2 \sigma=$ $b, 2 \rho_{2}+(\sigma-1) a_{2}=0$. Let us suppose the former, and that

$$
U=1+\Delta, \quad \alpha=1+\nu, \quad|\nu|,|\Delta| \ll 1 .
$$

It follows that (assuming $\gamma<2$ )

$$
\begin{aligned}
& u_{1} \sim 1-\gamma+\frac{\gamma \Delta}{2-\gamma}-\frac{2 \nu \gamma(1-\gamma)}{(2-\gamma)} \cdots, \\
& u_{2} \sim-1+\frac{\left[-(\Delta+\nu \gamma)+\left\{(\Delta+\nu \gamma)^{2}+\nu^{2} \gamma(2-\gamma)\right\}^{1 / 2}\right]}{2-\gamma}, \\
& u_{3} \sim-1+\frac{\left[-(\Delta+\nu \gamma)-\left\{(\Delta+\nu \gamma)^{2}+\nu^{2} \gamma(2-\gamma)\right\}^{1 / 2}\right]}{2-\gamma},
\end{aligned}
$$

hence

$$
\begin{aligned}
& k^{2} \sim 1-\frac{2\left\{(\Delta+\nu \gamma)^{2}+\nu^{2} \gamma(2-\gamma)\right\}^{1 / 2}}{(2-\gamma)^{2}} \\
& U \sim 1+\Delta \\
& c \sim 1-\delta
\end{aligned}
$$

where we denote

$$
\delta=\frac{2(2-\gamma)}{\ln \left[8(2-\gamma)^{2} /\left\{(\Delta+\nu \gamma)^{2}+\nu^{2} \gamma(2-\gamma)\right\}^{1 / 2}\right]}
$$

notice that $\Delta, \nu \ll \delta$. It follows that

$$
U-c \sim \delta
$$


so that

$$
\begin{gathered}
B \sim 1-\left(\frac{2 \sigma-b}{b}\right) \delta \cdots, \\
\Gamma=G / B \sim a_{2}\left[1+\left(\frac{2 \sigma-b}{b}\right) \delta \cdots\right],
\end{gathered}
$$

but also from (3.5),

$$
\Gamma \sim a_{2}\left[1+\delta\left\{\frac{2 \rho_{2}}{(\sigma+1) a_{2}}+\frac{\sigma-1}{\sigma+1}-\left(\frac{2 \sigma-b}{b}\right)\right\} \cdots\right]
$$

it follows that

$$
2\left(\frac{2 \sigma-b}{b}\right)=\frac{2 \rho_{2}}{(\sigma+1) a_{2}}+\frac{\sigma-1}{\sigma+1}
$$

Also,

$$
d \sim \frac{2}{b+2}\left[\rho_{2}-\frac{1}{3}(1-b) a_{2}\right]\left[\rho_{2}-a_{2}+\frac{1}{(b+2) \lambda}\right]+O(\delta),
$$

and (3.6) becomes

$$
2 \lambda-1+d \sim \frac{1}{3}+O(\delta)
$$

Thus as $\delta \rightarrow 0, \lambda$ satisfies

$$
\lambda+\frac{\left[\rho_{2}-(1-b) a_{2} / 3\right]}{(b+2)}\left[\rho_{2}-a_{2}+\frac{1}{(b+2) \lambda}\right]=\frac{2}{3} .
$$

We denote the corresponding value(s) of $\lambda$ by $\lambda_{h}$, etc., and observe that for given $a_{2}$, (3.20) determines $\rho_{2}$ as a quadratic function of $\lambda$, so that there could be in general five such values.

The above results suggest that when $\lambda \rightarrow \lambda_{h}$, the equilibrium solutions are such that the underlying fast oscillations $(\theta)$ can approach the saddle point $\theta=\pi$, and hence that a homoclinic explosion can take place at such a parameter value. It is of interest to study the case of Fowler et al. [1], where (because of the model derivation), $e$ and $r_{2}$ are related by $e=3 r_{2}$. In terms of $a_{2}$ and $\rho_{2}$, this implies $\rho_{2}=\sigma a_{2} / 3 ;(3.20)$ and (3.23) are then (if $a_{2} \neq 0$ )

$$
(\sigma+1)\left(\frac{2 \sigma-b}{b}\right)=\frac{\sigma}{3}+\frac{\sigma-1}{2}
$$

and

$$
\sigma+1+\frac{1}{3} a_{2}[\sigma+b-1]\left[\frac{1}{\sigma+1}-a_{2}\left(1-\frac{\sigma}{3}\right)\right]=\frac{2}{3}(b+2) .
$$

For a given value of $b,(3.24)$ determines values of $a_{2}$ and $\sigma$, and a corresponding "homoclinic" curve in $\left(r_{1}, r_{2}\right)$ space as

$$
r_{2} \approx\left\{\sqrt{\sigma} a_{2} / 3\right\} r_{1}^{1 / 2} \text {. }
$$

The relation (3.25) is a prediction capable of numerical validation. Since homoclinic orbits near these values are presumably unstable, such a prediction must await confirmation by development of a special torus-following program. Nevertheless, the parabolic 
form of (3.25) ties in nicely with the apparent parabolic shape of curves $B$ and $C$ in Fig. 3 of Fowler et al. [1]. In particular, we might predict curve $B$ if we were able to do linear stability analysis of the fixed points in the $(B, D, G)$ plane; this seems prohibitively difficult, however.

The "Hopf" bifurcation of the limit cycle is much simpler. The critical $r_{1}\left(=r_{1 c}^{\prime}\right)$ is given in this case by Fowler et al. [1], and we find that, if $e=k r_{2}$,

$$
r_{1 c}^{\prime} \sim\left[\frac{(k+1)(k-\sigma)}{(\sigma+1)^{2}}+\frac{2\{2 \sigma+(\sigma-1) k\}}{b(\sigma-b-1)(\sigma+1)}\right] r_{2}^{2} ;
$$

in terms of $\rho_{2}$, this gives the critical value

$$
\rho_{2 c}^{\prime} \sim 3\left[\frac{b(1-b)}{21+2 b(1-b)}\right]^{1 / 2},
$$

where we also put $e=3 r_{2}(k=3)$ and $\sigma=2$. For $b=0.8$, this is $\rho_{2 c}^{\prime} \sim 0.26$, or $r_{2} \sim$ $0.18 \sqrt{r_{1}}$, more in line with (though still greater than) curve $C$ in Fig. 3 of Fowler et al. [1].

Form of the trajectories in the original variables. Returning to $(\xi, \eta, \zeta)$ space, we see that the steady state toroidal solution may be written in the form

$$
\boldsymbol{\xi}=R(\phi) \mathbf{v},
$$

where $\xi=\left(\xi_{R}, \xi_{I}, \eta_{R}, \eta_{I}, \zeta\right)^{T}, \mathbf{v}=\left(-\dot{\theta},-\left(G+a_{2} \cos \theta\right) / \sin \theta, B \sin \theta, 0,1-B \cos \theta\right)^{T}$ is periodic of period $T=2 K\left[2 / B\left(u_{1}-u_{3}\right)\right]^{1 / 2}$, and $R$ is the rotation matrix discussed earlier; this is in the same form as (1.12), but $\phi$ is not singly periodic, since although $\dot{\phi}$ given by (2.10) has period $T$, its mean is not necessarily zero. If we define

$$
\overline{\dot{\phi}}=\tilde{\omega}, \quad \phi=\tilde{\omega} t+\tilde{\phi},
$$

then $\tilde{\phi}$ is periodic of period $T$, and we may write (3.28) in the form

$$
\boldsymbol{\xi}=R(\tilde{\omega} t) \mathbf{u}(\tilde{\Omega} t),
$$

where

$$
\mathbf{u}(\tilde{\Omega} t)=R(\tilde{\phi}) \mathbf{v}, \quad \tilde{\Omega}=2 \pi / T,
$$

and $\mathbf{u}$ and $R$ have frequencies $\tilde{\Omega}$ and $\tilde{\omega}$, respectively. This shows that (1.12) is relevant at both high and low $r_{1}$, and we can safely conjecture that it is valid in between, as well. The frequencies at high $r_{1}$ are given by

$$
\tilde{\Omega}=\frac{\pi}{K}\left(\frac{B\left(u_{1}-u_{3}\right)}{2}\right)^{1 / 2}, \quad \tilde{\omega}=\left[\overline{\frac{G \cos \theta+a_{2}}{\sin ^{2} \theta}}\right],
$$

from (2.10). $\tilde{\omega}$ can be calculated, and we find

$$
\tilde{\omega}=\frac{1}{2 K}\left[\frac{\left(a_{2}-G\right)}{1+u_{1}} \Pi\left(\frac{u_{1}-u_{2}}{1+u_{1}}, k\right)+\frac{\left(a_{2}+G\right)}{1-u_{1}} \Pi\left(-\frac{\left(u_{1}-u_{2}\right)}{1-u_{1}}, k\right)\right],
$$

where $\Pi$ is the complete elliptic integral of the third kind. We can evaluate $\tilde{\omega}$ as $G \rightarrow 0$ (i.e., $a_{2}, \rho_{2} \rightarrow 0$ ): as $\gamma=G^{2} / 2 B \rightarrow 0$, we find

$$
\begin{aligned}
& u_{1} \sim 1-\frac{\gamma(1+\alpha)^{2}}{2(1+U)}, \\
& u_{2} \rightarrow-U, \\
& u_{3} \rightarrow-1,
\end{aligned}
$$


provided $U<1$; from the previous discussion, this is the relevant parameter range for describing the stable torus as $\rho_{2} \rightarrow 0$. As $\gamma \rightarrow 0, \Pi\left(\left(u_{1}-u_{2}\right) /\left(1+u_{1}\right), k\right)$ is bounded, but we find $\Pi\left(-\left(u_{1}-u_{2}\right) /\left(1-u_{1}\right), k\right) \rightarrow \infty$, and using (3.34) and the definition of $\gamma$, we find

$$
\tilde{\omega} \sim \frac{\pi}{2 K}\left[\frac{B(1+U)}{u_{1}-u_{2}}\right]^{1 / 2} ;
$$

comparing this with (3.32), and using (3.34), we find that indeed

$$
\Omega \sim \frac{\pi}{K} \sqrt{B}, \quad \tilde{\omega} \sim \frac{\pi}{2 K} \sqrt{B} \sim \frac{1}{2} \tilde{\Omega},
$$

as $\rho_{2} \rightarrow 0$, thus bearing out the observations of $\S 1$.

Anomalous solutions. At least for $\rho_{2}$ small enough, we can expect that the $U>1$ (Hopf bifurcating) torus is unstable, and the $U<1$ torus is stable. It does not seem possible to discuss the stability of the fixed points in any serious manner, and in any case, bifurcations in the $(B, D, \Gamma)$ phase space would hardly correspond to perioddoublings observed at small $\rho_{2}$ and finite (if large) $r_{1}$ : such period-doubling is probably beyond the range of our analysis. In fact, if we believe (following Sparrow [21]) that the coalescence of $U<1$ and $U>1$ fixed points at $\lambda=\lambda_{h}$ (from (3.23)) represents the approach to a homoclinic torus, then we might correspondingly expect the explosion of an infinite number of tori in the complex case at this value of $\lambda$. Since all these orbits at high $r_{1}$ could be equally well described by the averaging procedure, we might conclude that to $O(\varepsilon)$ they are indistinguishable, and that the line of orbits (dotted in Fig. 4 for $\left.\rho_{2} \neq 0\right)$ "really" represents a thin $O(\varepsilon)$-wide sheath of orbits which would disappear in period-doubling windows as $r_{1}$ decreases (for $\rho_{2}=0$, one can imagine an $\varepsilon$-axis in Fig. 4 vertically out of the page: the periodic orbits at fixed $U$ would mushroom out of the page as $\varepsilon$ increases from zero and later disappear via coalescence). Presumably a higher-order averaging theory might resolve these windows.

Sparrow also discusses the problem of the invariant line $G=a_{2}, B=D$; as a trajectory tends towards this line, $k \rightarrow \infty$, and the method of averaging formally breaks down when $\varepsilon K \sim 1$. With $U$ and $\alpha$ defined by (3.12), one finds

$$
K \sim \frac{2-\gamma}{\delta}
$$

as $\delta \rightarrow 0$, where $\delta$ is given by (3.15). It follows that averaging breaks down when

$$
B-D \sim \exp [-O(1 / \varepsilon)]
$$

which implies

$$
\zeta \sim O(1), \quad \xi, \eta \sim \exp [-O(1 / \varepsilon)]
$$

As $\delta \rightarrow 0$, we use the results and definitions of (3.12) to (3.15), and find that, to $O(\delta)$,

$$
\begin{aligned}
& D^{\prime} \sim b(1-B)-(2 \sigma-b) \delta B, \\
& B^{\prime} \sim b(1-B)-\delta\left[b-a_{2} \rho_{2}+\frac{2}{3}(1+\gamma)(1-b) B\right], \\
& \Gamma^{\prime} \sim+a_{2} b(1-B)+\delta B\left[2 \rho_{2}-a_{2}(\sigma+1-b)\right] .
\end{aligned}
$$

(3.40) is derived on the basis that $B-D$ and $\Gamma-a_{2} B$ are both small, and is clearly consistent with this assumption. Notice that $\delta$ given by (3.15) is not constant in (3.40), but is itself a function of $B, D$, and $G$. So long as $\delta \rightarrow 0$, we rewrite (3.40), using 
$D-B=B \Delta, \Gamma-a_{2} B \approx-\nu a_{2} B$, as

$$
\begin{aligned}
& B^{\prime} \sim b(1-B)+O(\delta), \\
& (B \Delta)^{\prime} \sim \delta\left[b-a_{2} \rho_{2}+\left\{\frac{2}{3}(1+\gamma)(1-b)-(2 \sigma-b)\right\} B\right], \\
& (B \nu)^{\prime} \sim \delta\left[-\left(b-a_{2} \rho_{2}\right)+\left\{\sigma+1-b-2 \rho_{2} / a_{2}-\frac{2}{3}(1+\gamma)(1-b)\right\} B\right] .
\end{aligned}
$$

We can anticipate that the logarithmic dependence of $\delta$ on $\nu$ and $\Delta$ will enable these variables to go to zero in a finite time. For simplicity, consider first the real case, in which $\gamma=a_{2}=\rho_{2}=\Gamma=0$, so that

$$
\delta=4 / \ln (32 /|\Delta|)=4 \ln (1 /|\Sigma|)
$$

where we define $\Sigma=\Delta / 32$. ignoring $\Delta B^{\prime}=o(\delta),(3.41)$ is then

$$
\begin{aligned}
& B^{\prime} \sim b(1-B), \\
& \Sigma^{\prime} \sim-\frac{\mu}{24}\left[\ln \frac{1}{|\Sigma|}\right]^{-1},
\end{aligned}
$$

where

$$
\mu=6 \sigma-b-2-3 b / B
$$

Over time scales $|t| \ll 1, B$ changes very little, and we can consider it approximately constant. $(3.43)_{2}$ then integrates to

$$
\Sigma[1+\ln (1 /|\Sigma|)]=-\mu t / 24
$$

plus a constant which we have put to zero. In this case, (3.45) shows that $\Sigma \rightarrow 0$ in small (finite) time, and since $D / B=U=1+32 \Sigma$, then $D>B$ for $t>0$ if $\mu<0, D<B$ for $t<0$ : that is, trajectories reach and leave the line $D=B$ in the direction of increasing $D / B$ if $\mu<0$ (and in the direction of decreasing $D / B$ if $\mu>0$ ). These conclusions were found by Sparrow [21].

To extend the results to $\rho_{2} \neq 0$, we suppose that the equations (3.41) are to be integrated over a small range of $|t|$ (if we expect both $\Delta$ and $\nu$ to tend to zero in finite time). We can consistently neglect the small terms $\Delta B^{\prime}, \Delta \nu^{\prime}$. Then

$$
\begin{aligned}
& \Delta^{\prime} \sim \delta\left[\left(b-a_{2} \rho_{2}\right) / B+\frac{2}{3}(1+\gamma)(1-b)-(2 \sigma-b)\right], \\
& \nu^{\prime} \sim \delta\left[-\left(b-a_{2} \rho_{2}\right) / B+\sigma+1-b-2 \rho_{2} / a_{2}-\frac{2}{3}(1+\gamma)(1-b)\right],
\end{aligned}
$$

and we deduce $\Delta \approx c_{0} \nu+c_{1}$. If $c_{1} \neq 0$, then $\delta \neq 0$, and $\Delta, \nu$ will pass through zero, but not simultaneously, and thus not intersect the invariant line. However, in every (small) two-dimensional neighborhood of this line (for fixed $B$ ), there is a one-parameter family of initial points for (3.46) for which $c_{1}=0$. In this case, $\Delta=c_{0} \nu$, with

$$
\begin{aligned}
c_{0} & =\frac{\left[\left(b-a_{2} \rho_{2}\right) / B+\frac{2}{3}(1+\gamma)(1-b)-(2 \sigma-b)\right]}{\left[-\left(b-a_{2} \rho_{2}\right) / B+\sigma+1-b-2 \rho_{2} / a_{2}-\frac{2}{3}(1+\gamma)(1-b)\right]}, \\
& =c_{T} / c_{B}, \quad \text { say, }
\end{aligned}
$$

and we have

$$
\ln \left[\frac{1}{|\Sigma|}\right] \Sigma^{\prime} \sim \frac{\left[\left(c_{0}+\gamma\right)^{2}+\gamma(2-\gamma)\right]^{1 / 2}}{4(2-\gamma)} c_{B}
$$

where

$$
\Sigma=\frac{\left\{\left(c_{0}+\gamma\right)^{2}+\gamma(2-\gamma)\right\}^{1 / 2}}{8(2-\gamma)^{2}} \nu
$$


The solution of $(3.48)$ is $\Sigma[1+\ln (1 /|\Sigma|)]=t \times($ RHS of (3.48)) and thus $\Sigma$, and hence $\nu$ and $\Delta$ will reach zero in finite time. Thus trajectories with the right initial values will approach and leave the invariant line in the direction of increasing $D / B(=U=$ $1+\Delta$ ) if $\Delta$ increases with $t$, i.e. if $c_{T}>0$, or

$$
6 \sigma-(1-2 \gamma) b-2(1+\gamma)-3\left(b-a_{2} \rho_{2}\right) / B<0,
$$

and they approach and leave the invariant line in the direction of increasing $G(\approx$ $\left.a_{2}(1-\nu)\right)$ if $\nu$ decreases with $t$, i.e. if $c_{B}<0$, i.e.

$$
3\left(\sigma+1-2 \rho_{2} / a_{2}\right)-(1-2 \gamma) b-2(1+\gamma)-3\left(b-a_{2} \rho_{2}\right) / B<0 .
$$

Along the line $B-D=G-a_{2}=0$, there is a genuine nonuniqueness of the averaged equations (2.26). Trajectories can reach and leave this line in finite time without crossing it, and also apparently travel along it. Of course the averaging method breaks down at this stage, but we can partially understand what happens by recourse to Fowler and McGuinness [2]. As $B \rightarrow D \rightarrow 0, G \rightarrow a_{2}$, so $\xi, \eta \rightarrow 0$ and $\zeta$ begins an exponential decay towards zero $(B \rightarrow 1)$; this corresponds to the slow phase of the relaxation oscillation which occurs when $\rho_{2}=0, \sigma \sim r_{1} \rightarrow \infty$. Thus we should expect an analysis of the different asymptotic structure which is relevant when $B=D$ to determine the time after arrival at which the trajectory is kicked off the line, due to the eventual exponential growth of $\xi$ and $\eta$ as $\zeta$ becomes small. In principle, such anomalous trajectories could join up to give anomalous periodic orbits: these are more fully discussed by Sparrow. We only point out that as $\sigma$ increases, we might expect such anomalous orbits to become stable, and an interesting question is then, how does one get from the parameter regime $r_{1} \sim \sigma \gg 1$ to the regime $r_{1} \gg 1, \sigma \sim 1$ ?

4. Conclusions. Our main result is that the torus in the complex Lorenz equation can indeed be written in the rotational form (1.12). Specifically, we have the following bifurcation structure:

$$
\text { fixed point } \begin{aligned}
\mathbf{x}=0 & \rightarrow \text { limit cycle } x=R(\omega t) \mathbf{x}_{0} \\
& \rightarrow \text { torus } \mathbf{x}=R(\tilde{\omega} t) \mathbf{u}(\tilde{\Omega} t)
\end{aligned}
$$

as the parameter $r_{1}$ increases. These descriptions are global, rather than local. The rotational forms (4.1) clearly stem from the rotational invariance of the system, and we can at least pose the questions, can one prove the forms (4.1) for the complex Lorenz system, or for more general systems (one thinks in this context of Taylor vortices in rotating Couette flow [16]). Evidence for the rotational torus as in (4.1) comes from local bifurcation analysis (Fowler et al. [1]), is corroborated by an asymptotic analysis at high $r_{1}$, and further substantiated by numerical experiments at intermediate values of $r_{1}$. The high $r_{1}$ analysis further suggests that a homoclinic explosion of tori takes place at a critical value of $\lambda \sim \lambda_{h}$ as $r_{1} \rightarrow \infty$, and thus that the numerically observed torus may undergo period-doubling bifurcation cascades as $r_{2} \rightarrow 0$ at finite $r_{1}$, as observed.

Another facet of the torus is that as $r_{2} \rightarrow 0, r_{1} \rightarrow \infty$, the rotating frequency $\tilde{\omega}$ tends to a value which is half that of the underlying cycle. We predict this analytically, as well as observing it numerically (in spectral plots): in this way the torus reduces to a limit cycle at $r_{2}=0$, as it must, for other reasons, being a dissipative system.

In the caption to Fig. 4 , we have already noted that if $|1-B| \ll 1$, then we can define the trajectories in $(B, D)$ space for the real case by the approximate relation 
(since $\delta \ll \Delta$, from $(3.15)$ ),

$$
|\Delta| \sim 32 \exp [-4(2 \sigma-b) / b s],
$$

where as before $\Delta \sim$ distance from the $B=D$ axis, and $s \sim 1-B$ is proportional (a factor of $\sqrt{2}$ ) to the distance along the $B=D$ axis from $B=D=1$. The interesting thing about (4.2) is that, although formally $|\Delta| \ll 1$ only if $s \ll 1$ when $\sigma, b \sim 1$, nevertheless $s$ is only logarithmically small, and so can be numerically of reasonable size. In other words, equilibria can correspond to near-homoclinic orbits in quite a large neighborhood of $\lambda_{h}$. Numerically, this means it may be hard to "get at" the homoclinic orbits. On the other hand, since averaging breaks down when $|\Delta| \sim \exp [-O(1 / \varepsilon)]$, we can expect anomalous orbits to appear when $|\Delta|$ is of this order. Thus if the equilibria are "close" enough to $B=1$ that (4.2) is valid, and $|\Delta| \sim \exp (-O(1 / \varepsilon))$, then we can expect the real system to be able to maintain stable anomalous orbits. From (4.2), this is when

$$
s \sim 4 \varepsilon[2(\sigma / b)-1],
$$

and is generally $\ll 1,(s \rightarrow 0$ as $\varepsilon \rightarrow 0)$, unless $\sigma / b$ becomes large. If $s$ is indeed small, then the above discussion implies that for $\lambda>\lambda_{h}$, anomalous orbits will be an unstable, transient feature. However, they will become of global relevance when $s \sim O(1)$ : from (4.3), this is when

$$
b / \sigma \sim \varepsilon=1 / \sqrt{r_{1} \sigma}
$$

and implies that in a distinguished limit given by (4.4), or particularly when $r_{1} \sim \sigma \gg 1$, $b \sim 1$, this style of chaos will prevail. For the real Lorenz system $\left(\rho_{2}=0\right)$, this parameter regime has been studied by Fowler and McGuinness [2].

As a last comment, let us admit that the study of systems such as the complex Lorenz equations is only ultimately "justified" if it sheds light on behaviors in a real physical system. Apart from the actual derivation of the present system in models of (for example) baroclinic instability, we may point out the resemblance of the bifurcation behavior here to that in rotating Couette flow. The study of the Lorenz equations has now led in Sparrow's work to the recognition that a new type of bifurcation, associated with the occurrence of a homoclinic orbit, can give rise to strange behavior unrelated (at least parametrically) to any Hopf bifurcation in the system. If we think of the transition to turbulence in plane Poiseuille flow at $\mathrm{Re} \sim 1000$, and the (unrelated) subcritical Hopf bifurcation at $\mathrm{Re} \approx 5772$, one may suppose that analogies in simple systems may have relevance in real fluid problems. One can also draw interesting (and direct) parallels between the behavior of the real Lorenz equations at high $r_{1}$ and $\sigma$ and Howard's [5] schematic description of thermal turbulence, and we can therefore hope that the development of techniques and results for systems of ordinary differential equations may eventually bear fruit in the field of fluid mechanics.

Acknowledgments. We would like to acknowledge discussions with John Gibbon, and particularly to thank Colin Sparrow for providing a pre-publication view of his book [21] on the Lorenz equations. As will be seen, the development of $\S 3$ is largely based on Chapter VII of his book, and the style of piecing together a global analytic picture from bits of numerical and analytical evidence is very much his.

\section{REFERENCES}

[1] A. C. Fowler, J. D. GibBon And M. J. MCGuinness, The complex Lorenz equations, Physica, 4D (1982), pp. 139-163. 
[2] A. C. Fowler AND M. J. MCGuinness, A description of the Lorenz attractor at high Prandtl number, Physica, 5D (1982), pp. 149-182.

[3] J. D. GibBon, Dispersive instabilities in nonlinear systems: the real and complex Lorenz equations, in Chaos and Order in Nature, H. Haken, ed., Springer-Verlag, Berlin, 1981, pp. 92-101.

[4] J. D. GibBon AND M. J. MCGuINNESs, The real and complex Lorenz equations in rotating fluids and lasers, Physica, 5D (1982), pp. 108-122.

[5] L. N. HowARD, Convection at high Rayleigh number, in Proceedings of the Eleventh International Congress of Applied Mechanics, H. Görtler, ed., Springer-Verlag, Berlin, 1966, pp. 1109-1115.

[6] — Nonlinear oscillations, Lectures in Applied Mathematics, Vol. 17, American Mathematical Society, Providence, RI, 1979, pp. 1-67.

[7] D. D. JOSEPH, The bifurcation of T-periodic solutions into nT-periodic solutions and TORI, in Synergetics-a Workshop, H. Haken, ed., Springer-Verlag, Berlin, 1977, pp. 39-50.

[8] J. Kevorkian And J. D. Cole, Perturbation Methods in Applied Mathematics, Springer-Verlag, 1981.

[9] S. Kogelman AND J. B. Keller, Asymptotic theory of nonlinear wave propagation, this Journal, 24 (1973), pp. 352-361.

[10] G. E. KUZMAK, Asymptotic solutions of nonlinear second order differential equations with variable coefficients, PMM, 23 (1959), pp. 515-526; Appl. Math. Mech., 23 (1959), pp. 730-744.

[11] E. N. Lorenz, Deterministic non-periodic flow, J. Atmos. Sci., 20 (1963), pp. 130-141.

[12] J. C. LuKE, A perturbation method for nonlinear dispersive wave problems, Proc. R. Soc. Lond., A 292 (1966), pp. 403-412.

[13] J. B. MCLAUghlin AND P. C. MARTIN, Transition to turbulence in a statically stressed fluid system, Phys. Rev., A12 (1975), pp. 186-203.

[14] E. OTT, Strange attractors and chaotic motions of dynamical systems, Rev. Mod. Phys., 53 (1971), pp. 655-671.

[15] J. P. POYET, Time-dependent convection, Thesis, Columbia University, New York, 1980.

[16] D. RAND, Dynamics and symmetry: predictions for modulated waves in rotating fluids, Arch. Rat. Mech. Anal., 79 (1982), pp. 1-37.

[17] M. RENARDY, Bifurcation from rotating waves, Arch. Rat. Mech. Anal, 79 (1982), pp. $49-84$.

[18] K. A. RobBins, A new approach to subcritical instability and turbulent transitions in a simple dynamo, Math. Proc. Camb. Phil. Soc., 82 (1977), pp. 309-325.

[19] - Periodic solutions and bifurcation structure at high $R$ in the Lorenz model, this Journal, 36 (1979), pp. 457-472.

[20] N. V. RoshCHIN, Unsafe stability boundaries of the Lorentz (sic) model, Prikl. Math. Mekh., 42 (1978), pp. 950-953; in translation: Appl. Math. Mech., 42 (1978), pp. 1038-1041.

[21] C. SPARROW, The Lorenz equations: bifurcations, chaos, and strange attractors, Lecture Notes in Applied Mathematics 41, Springer, Berlin, 1982. 